\title{
Quantum networks: topology and spectral characterization
}

\author{
Vesna Berec ${ }^{1,2 a}$ \\ ${ }^{1}$ University of Belgrade, St. Trg 16, 11000, Belgrade, Serbia \\ ${ }^{2}$ Institute of Nuclear Sciences Vinca, P.O. Box 522, 11001 Belgrade
}

\begin{abstract}
To utilize a scalable quantum network and perform a quantum state transfer within distant arbitrary nodes, coherence and control of the dynamics of couplings between the information units must be achieved as a prerequisite ingredient for quantum information processing within a hierarchical structure. Graph theoretic approach provides a powerful tool for the characterization of quantum networks with non-trivial clustering properties. By encoding the topological features of the underlying quantum graphs, relations between the quantum complexity measures are presented revealing the intricate links between a quantum and a classical networks dynamics.
\end{abstract}

\section{Introduction}

The need of complex networks quantum representation developed rapidly proceeding an intensive research field of quantum computing two decades ago [1]. Ranging from first concepts of an intersection between complex network systems and quantum dynamics [2], number of studies introduced the notions of 'quantum neural network' [3,4] and spin networks [5-7]. As the prime motive in latter complex architectures, utilization of linear, unitary dynamics of quantum computing [1] into highly nonlinear and complex framework of network systems dynamics, capturing at the same time the interrelations between the individual elements of systems and complete databases, proved to be a highly demanding task. Presented graph theoretical approach relies on a master equation intersecting between quantum coherent dynamics and the classical diffusion, where linearized dynamical process on a complex network system is encoded into the simplicial set matrix of the underlying network and related to its topological properties. Spin networks [5-10] are purely combinatorial structures, represented by simplicial complexes with edges labeled by numbers $j=0,1 / 2,1,3 / 2$, etc. These numbers stand for total angular momentum or "spin". The imposed condition for spin network architecture is that three edges meet at each vertex, with the corresponding spins: $j_{1}, j_{2}, j_{3}$, adding up to an even integer and satisfying the triangle inequality. These rules are motivated by the quantum properties of angular momentum: if we combine a component with spin $j_{1}$ and a component with spin $j_{2}$, the spin $j_{3}$ of the unit system satisfies exactly latter constraint. In such setting, given that $\mathbb{F}$ is a general field, a spin network represents quantum states of

\footnotetext{
a e-mail: bervesn@gmail.com
} 
defined by tensor product states: $\mathbb{F}$-geometry on $d=3+1$ dimensional space

$$
H_{j_{1} j_{2} j_{3} j_{4}} \equiv \otimes_{i=1}^{4} H_{j i}, H_{\perp} \equiv \underset{\{J\}}{\oplus} H_{\{J\}}^{0}
$$

where $\{J\}$ runs over the set of ordered 4-tuples of integers or half-integers such that $H_{\{J\}}^{0}$ is the nonempty complex obtained from the $n$-skeleton $H^{n}$, constructed from $H^{n-1}$ by attaching $n$-simplexes (adjoined to a simplicial complex $K$ ) via maps $\phi: K^{n-1} \rightarrow H^{n-1}$. In the PR model [5] a partition function is defined for a given 3-dimensional simplicial complex (by deforming $\mathrm{SU}(2)$ to a quantum group [8], where the partition function depends only on the topology of the manifold which is triangulated by the simplicial complex) by means of the following: to each edge of the complex is associated a spin (i.e., an irreducible unitary $\mathrm{SU}(2)$ representation, determined only by its dimension $d \equiv 2 j+1$ ).

In particular case, we are interested in the homomorphisms of the simplicial $q$-th homology group which represents the free abelian group generated by the $q$-cycles, and their induced mapping on the stabilizer group $\left(S_{G}\right)$ basis. Assuming that $\Gamma$ and $S_{G}$ are free abelian groups with bases $g_{1}, \ldots, g_{n}$ and $g_{1}^{\prime}, \ldots, g_{m}^{\prime}$, respectively, if $f: \Gamma \rightarrow S_{G}$ is a homomorphism, then $f\left(g_{j}\right)=\sum_{i=1}^{m}(-1)^{i} \lambda_{i j} g_{i}^{\prime}$ for unique integers $\lambda_{i j}$, where the parity of any transposition is -1 . More general, giving that $K$ is an oriented simplicial complex, and $S_{G}$ is an abelian group, then for non-negative integer $q$, to each $(q+1)$-tuple $\left(x_{0}, x_{1}, \ldots, x_{q}\right)$ of vertices spanning a simplex $\sigma_{q}(K)$, there corresponds an element $\alpha\left(x_{0}, x_{1}, \ldots, x_{q}\right)$ of $S_{G}$ defining a homomorphism $\alpha: C_{q}(K) \rightarrow S_{G}$, where $C_{q}(K)$ denotes the corresponding chain group, i.e., finitely generated abelian group on the oriented simplices.

\section{Results and Discussion}

Let $V$ be the vertex set and $e_{1}, e_{2}, \ldots, e_{n}$ be the sequence of edges on $V \times V$, connected along a path from a point $a$ to a point $b$ on the surface $S$, given by: $e_{i}=P_{i} P_{i+1}, P_{1}=a, P_{n+1}=b$, where distinct edges possess orientation which coincides with the path direction. Then the path can be associated to the 1-chain: $e_{1}+e_{1} \cdots+e_{n}$. A linear transformation of the 1-chain module is associated by each group element action $g \in \Gamma$ which permutes the edges in either the successive mirror or the dual tiling, defining: $\alpha_{1} e_{1}+\alpha_{2} e_{2}+\cdots \alpha_{n} e_{n} \rightarrow \alpha_{1} g e_{1}+\alpha_{2} g e_{2}+$ $\cdots \alpha_{n} g e_{n}$. In general, the $\Gamma$-action commutes with the boundary operator, i.e., $\partial g n=g \partial n$ for every chain, where:

$g Z_{n}(S ; \mathbb{R})=Z_{n}(S ; \mathbb{R})=\operatorname{ker}_{n}: C_{n}(S ; \mathbb{R}) \rightarrow C_{n-1}(S ; \mathbb{R})$,

$g B_{n}(S ; \mathbb{R})=B_{n}(S ; \mathbb{R})=\operatorname{im} \partial_{n}: C_{n+1}(S ; \mathbb{R}) \rightarrow C_{n}(S ; \mathbb{R})$,

showing that distinct elements of $\Gamma$ map homology classes to homology classes, yielding a linear action of $\Gamma$ on $H_{n}(S ; \mathbb{R})=\frac{Z_{n}(S ; \mathbb{R})}{B_{n}(S ; \mathbb{R})}$. Then, a corresponding vertex set $V$ represents a submodule for $V \subseteq H_{n}(S ; \mathbb{R})$ which is $\Gamma$-invariant or a $\Gamma$-submodule if $g V=V, \forall g \in \Gamma$. Such action of group $\Gamma$ on the homology group of a chain complex is known as the homology representation.

Let $\Gamma$ be a group, where $S \subseteq \Gamma$ is a generator subset. Let $\bar{S}$ be a set of inverses of $S$ with $A=S \sqcup \bar{S}$. Then, an underlying graph [16,17] of spin network $G=G(\Gamma, S)$ is established by connecting vertices $g, h \in V_{G}$, where the set $V_{G} \subset \Gamma$, by establishing edge in $A$ under condition:

$$
\left(g, h \in V_{G}\right)= \begin{cases}1, & \text { if } \mathrm{g}^{-1} h \in A \\ 0, & \text { otherwise }\end{cases}
$$


That is, for distinct $g \in \Gamma$ and $a \in A$ there is an edge relating $g$ to $g a$. In particular, the directed edge from $g$ to $g a$ is defined as the element $a$.

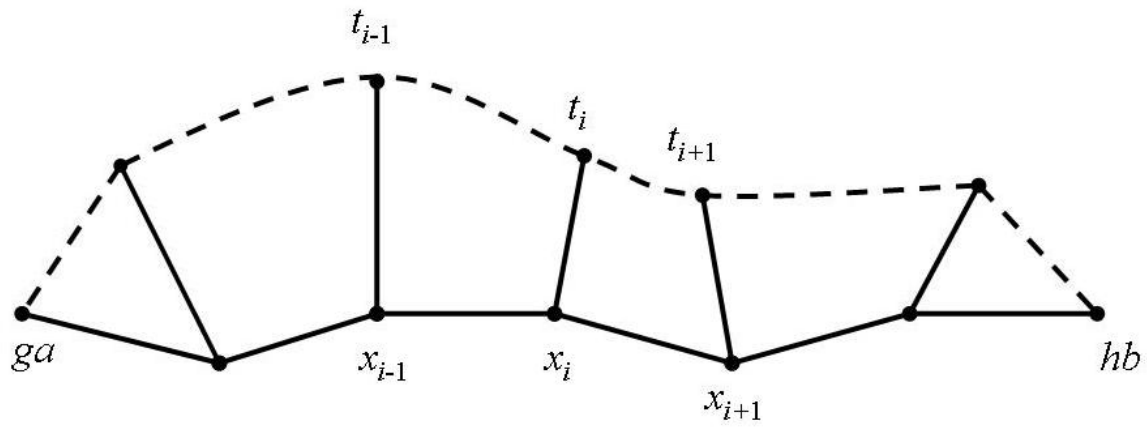

Figure 1. Construction of a spin network by the union of the set of flat connections which can be defined over the multiply connected manifolds [18], given by unit intervals of a finite set of curves crosshatching only at their endpoints of the metric space [19].

Given any $g, h \subseteq \Gamma$, let $\alpha \subseteq V_{G}$ be a geodesic connecting ga to a point $h b$, by selecting a sequence of points, $g a=x_{0}, x_{1}, \ldots, x_{n}=h b$, see figure 1 , along $\alpha$, such that $d\left(x_{i}, x_{i+1}\right) \leqslant 1, \forall i$. For each $i, g_{i} \in V_{G}$ are selected so that $\alpha:[a, b] \rightarrow[0,1]$. Given a metric space $(M, d)$, let $I \subseteq \mathrm{R}$ be an interval. A path which denotes unit interval (geodesic) is

$\gamma: I \rightarrow\{M|d(\gamma(t), \gamma(\tau))=| t-\tau \mid, \forall(t, \tau) \in I\}$. Assuming $\gamma:[a, b] \rightarrow M$ is an arbitrary path, its length is represented as:

$$
\sup \left\{\sum_{i=1}^{n} d\left(\gamma\left(t_{i-1}\right), \gamma\left(t_{i}\right)\right) \mid a=t_{0}<t_{1}<\cdots<t_{n}=b\right\} .
$$

Let $X_{\sigma}$ and $X_{\sigma^{\prime}}$ be subspaces of $X$ such that the covering dimension of simplexes $\sigma, \sigma^{\prime}$ is maximal covering of $X$. Let $\gamma_{i}: X_{\sigma \sigma^{\prime}} \rightarrow X_{\gamma}$ and $\gamma_{j}: X_{\gamma} \rightarrow X$ be the inclusions, resulting that $h_{\gamma}: \Pi\left(X_{\gamma}\right) \rightarrow \Lambda$ are functors into a groupoid defining the commutativity relation $h_{\sigma^{\prime}} \Pi\left(i_{\sigma^{\prime}}\right)=h_{\sigma} \Pi\left(i_{\sigma}\right)$, i.e., a different path $\gamma$ gives the same result, where a unique functor $\lambda: \Pi(X) \rightarrow \Lambda$ is defined such that $h_{\sigma^{\prime}}=\lambda \Pi\left(j_{\sigma^{\prime}}\right), h_{\sigma}=\lambda \Pi\left(j_{\sigma}\right)$ as:

$$
\begin{array}{cll}
\Pi\left(X_{\sigma \sigma^{\prime}}\right) & \stackrel{\Pi\left(i_{\sigma}\right)}{\longrightarrow} & \Pi\left(X_{\sigma}\right) \\
\Pi\left(i_{\sigma^{\prime}}\right) \downarrow & & \Pi\left(j_{\sigma}\right) \\
\Pi\left(X_{\sigma^{\prime}}\right) & \stackrel{\Pi\left(j_{\sigma^{\prime}}\right)}{\longrightarrow} & \Pi(X)
\end{array}
$$

is a pushout in groupoid of the inclusions $X_{\sigma} \supset X_{\sigma \sigma^{\prime}} \subset X_{\sigma^{\prime}}$.

In particular, a path $\gamma:[a, b] \rightarrow X$ represents a morphism $[\gamma]$ in $\Pi\left(X_{\gamma}\right)$ from $\gamma(a)$ to $\gamma(b)$ if we arrange it with an increasing homeomorphism $\alpha:[a, b] \rightarrow[0,1]$. If $a=t_{0}<t_{1}<\cdots<$ $t_{n}=b$ then $\gamma$ establishes the composition of the morphisms $\left[\gamma \mid\left[t_{i}, t_{i+1}\right]\right]$. Let $\gamma: I \rightarrow X$ be a 
path and let $\sigma:\{0, \ldots, n\} \rightarrow\{0,1\} \mid \gamma\left(\left[t_{i}, t_{i+1}\right]\right) \subset X\left(\sigma_{i}\right)$, then there exists a decomposition in affine space: $0=t_{0}<t_{1}<\cdots<t_{n+1}=1$ such that: $\left[\gamma \mid\left[t_{i}, t_{i+1}\right]\right] \subset X\left(\sigma_{i}\right), i=0, \ldots, n$. The construction $\left[\gamma \mid\left[t_{i}, t_{i+1}\right]\right]$ as path $\gamma_{i}$ in $X_{\gamma_{i}}$, produces composition

$$
[\gamma]=\Pi\left(\sigma_{\gamma(n)}\right)\left[\gamma_{n}\right] \circ \cdots \circ \Pi\left(\sigma_{\gamma(0)}\right)\left[\gamma_{0}\right]
$$

If subdivision $\lambda$ exists, then $\lambda[\gamma]=h_{\gamma(n)}\left[\gamma_{n}\right] \circ \cdots \circ h_{\gamma(0)}\left[\gamma_{0}\right]$ is inclined by the homotopy composition of the path.

Let $h:(\mathcal{K} \rightarrow X$ be a homotopy of paths from $a$ to $b$. We consider edge-paths in the subsets of 3 -simplex $\left(\mathcal{K}_{3}\right)$ which path-connect coordinates $a=(000)$ and $b=(111)$, see figure 2 . These paths differ from $h_{\gamma(0)}$ and $h_{\gamma(1)}$ by composition with a constant interval. $h$ generates two paths in $\mathcal{K}$, which give the same result since they differ by a homotopy on subinterval which belongs to the subsets $\sigma_{i} \in \mathcal{K}_{3}, i=1, \ldots, 4$.

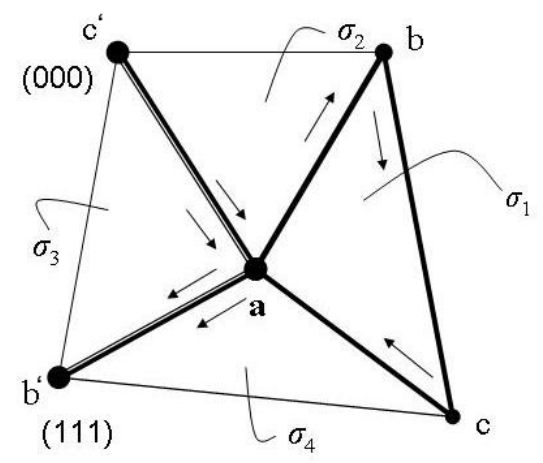

Figure 2. Two different paths (marked by thin and thick black lines) induce the following stabilizer generator sets on a base (a face) which belongs to incident simplexes (see theorem 2 and section 3 ): $\sigma_{1} \cap \sigma_{2}=\{\{a, b\}\} \rightarrow\{|000\rangle,|001\rangle,|110\rangle-|111\rangle\}$, $\sigma_{4} \cap \sigma_{1}=\{\{c, a\}\} \rightarrow\{|000\rangle,|101\rangle,|010\rangle-|111\rangle\}$, $\sigma_{3} \cap \sigma_{4}=\left\{\left\{a, b^{\prime}\right\}\right\} \rightarrow\{|000\rangle,|110\rangle,|001\rangle-|111\rangle\}$ $\sigma_{2} \cap \sigma_{3}=\left\{\left\{c^{\prime}, a\right\}\right\} \rightarrow\{|000\rangle,|010\rangle,|101\rangle-|111\rangle\}$.

Given a topological space $X$ representing the union of subsets $X_{\sigma}, X_{\sigma^{\prime}}$, general properties of $X$ encompassed from those of $X_{\sigma}, X_{\sigma^{\prime}}$, and $X_{\sigma \sigma^{\prime}}=X_{\sigma} \cap X_{\sigma^{\prime}}$ can be inferred from the theorem 2.

([15,16]) Let $K_{0}$ and $K_{1}$ be subspaces of simplicial complex $\mathcal{K}$ such that the covering dimension of simplexes $\sigma_{0} \in K_{0}, \sigma_{1} \in K_{1}$, is maximal covering of $X$. Considering $\gamma_{i}: K_{01}=K_{0} \cap K_{1} \rightarrow K_{\gamma}$ and $\gamma_{j}: K_{\gamma} \rightarrow X$ as inclusions, in particular, let $K_{0}, K_{1}, K_{01}$ be 
path connected with base $* \in K_{01}$. Then

$$
\begin{array}{lll}
\pi_{1}\left(K_{01}, *\right) & \stackrel{\pi\left(i_{1 *}\right)}{\longrightarrow} & \pi_{1}\left(K_{1}, *\right) \\
\pi_{1}\left(i_{0 *}\right) \downarrow & & \\
\pi_{1}\left(K_{0}, *\right) & \stackrel{\pi_{1}\left(j_{0 *}\right)}{\longrightarrow} & \pi_{1}\left(j_{1 *}\right) \\
& &
\end{array}
$$

is a pushout in topological space of the inclusions $K_{0} \supset K_{01} \subset K_{1}$, representing a fundamental group. Assuming that simplicial complex $\mathcal{K}$ is path connected and $z \in \mathcal{K}$, where $z=*$, then $r: \Pi(\mathcal{K}) \rightarrow \pi_{1}(\mathcal{K}, z)$ induces morphism compositions over the full subset $z$. For each $z \in \mathcal{K}$ exists a morphism such that $\mathrm{u}_{z}=\mathrm{id}, u_{y} \alpha u_{x}^{-1}$ where $\alpha: x \rightarrow y$, represented by:

$$
\begin{array}{ccc}
\Pi\left(K_{0}\right) & \longleftarrow \Pi\left(K_{01}\right) \longrightarrow & \Pi\left(K_{1}\right) \\
\downarrow r_{1} & \downarrow r_{01} & \downarrow r_{0} \\
\pi_{1}\left(K_{0}, *\right) & \leftarrow \pi_{1}\left(K_{01}, *\right) \rightarrow & \pi_{1}\left(K_{1}, *\right) .
\end{array}
$$

Precisely, restriction of $\mathcal{K}$ to subcomplexes: $\mathrm{K}_{01}, K_{0}, K_{1}$ and $X$ with a base point $z=*$, yields a commutative relation where morphisms in $\Pi(X)$ are respectively assigned by the composition of morphisms in $\Pi\left(K_{0}\right)$ and $\Pi\left(K_{1}\right)$, likewise, the group $\pi_{1}(X, *)$ is formed by the images of $j_{0} *$ and $j_{1} *$.

\section{Quantum network underlying architecture}

Graph state is represented in scope of the stabilizer formalism [21,22] via tensor products of Pauli operators $\sigma_{X}$ and $\sigma_{Z}$, whose composition and structure are based on the complexity of the underlying graph which can be seen as 1-dimensional simplicial complex. The stabilizers establish a group $\left(S_{G}\right)$ under multiplication, formed from $n$ generators $g_{i}$, associated to a number of vertices $x_{i}$ of the graph [23]. In particular, stabilizer generators are induced on the vertex set $V_{G}$ of a graph $G$ by the bijective mapping $\left(\Gamma\left(V_{G}\right), A\right) \rightarrow\left(S_{G}, \cdot\right)$, see section 3 , Eq. (3). Graph state is obtained by relating each vertex $x_{i} \in V_{G}$ with a stabilizer generator $g_{i}=\sigma_{X}^{i} \sigma_{Z}^{i j}$, where $g_{i}|G\rangle=|G\rangle, \forall i=1, \ldots, n$. The stabilizer generators [24-27] $g_{i}$ for $n$ graph state generate the complete Abelian stabilizer group $S_{G}$ of $|G\rangle$ with multiplication. The group $S_{G}$ consists of $2^{n}$ elements which uniquely represent a graph state

$$
|G\rangle=\left\{\sum_{i=1}^{2^{n}} \alpha_{i}\left|x_{i}\right\rangle=\sum_{i} \alpha_{i} S_{G}^{i}\left|x_{i}\right\rangle, \sum_{i}\left|\alpha_{i}\right|^{2}=1\right\} .
$$

The stabilizer group $S_{G}$ is formed from a set of $n-k$ generators $g_{1}, \ldots, g_{n-k}$, which: (a) commute; (b) are unitary and Hermitian; and (c) $g_{i}^{2}=I$. Each element of the stabilizer group $S_{G}$ can be expressed as a product of the generators as $S_{k}=g_{1}^{\alpha_{1}} \cdots g_{n-k}^{\alpha_{n-k}}, \quad \mathrm{~S}_{k} \in$ $S_{G}, \alpha_{i} \in\{0,1\}, i=1, \ldots, n-k$, where $S \subseteq G_{n}$ with $G_{n}$ denoting a corresponding Pauli group for $n$ qubit state.

Stabilizer code of length $n$ is represented by the fixed point set [27] $S_{k}=\{I, X, Y, Z\}$ of Pauli operators: 
$I=\left(\begin{array}{cc}0 & 1 \\ 1 & 0\end{array}\right), X \equiv \sigma_{X}=\left(\begin{array}{cc}0 & 1 \\ 1 & 0\end{array}\right), Y \equiv \sigma_{Y}=\left(\begin{array}{cc}0 & -i \\ i & 0\end{array}\right) Z \equiv \sigma_{Z}=\left(\begin{array}{cc}1 & 0 \\ 0 & -1\end{array}\right)$, $k=1, \ldots, n$ such that $S_{1}, S_{2}, \ldots, S_{k}$ are acting over $n$ qubits (i.e., over $\left.\left(\mathbb{C}^{2}\right)^{\otimes n}\right)$. When stabilizers $S$ are composed of elements $\left\{\sigma_{i}\right\}_{i=X, Y, Z}$ of $\{I, X\}^{\otimes n}$ and $\left\{\sigma_{i}\right\}_{i=X, Y, Z}$ of $\{I, Z\}^{\otimes n}$, it can be seen that $\left[\sigma_{i} \sigma_{j}\right]=2 i \varepsilon_{i j k} \sigma_{k}$ and $\left\{\sigma_{i} \sigma_{j}\right\}=2 \delta_{i j}$. Precisely, $I$ represents the identity matrix of size 2, $X$ denotes the Pauli matrix encoding the bit flip error and $Z$ denotes the Pauli matrix describing the phase error. The isomorphisms between $\{I, X\},\{I, Z\}$ and the vector space $\mathbb{F}_{2}$ makes possible establishing a connection between classical and quantum codes. On the basis of these isomorphisms, the stabilizers relate to binary vectors and the commutation relation corresponds to the orthogonality relation in $\mathbb{F}_{2}^{n}$.

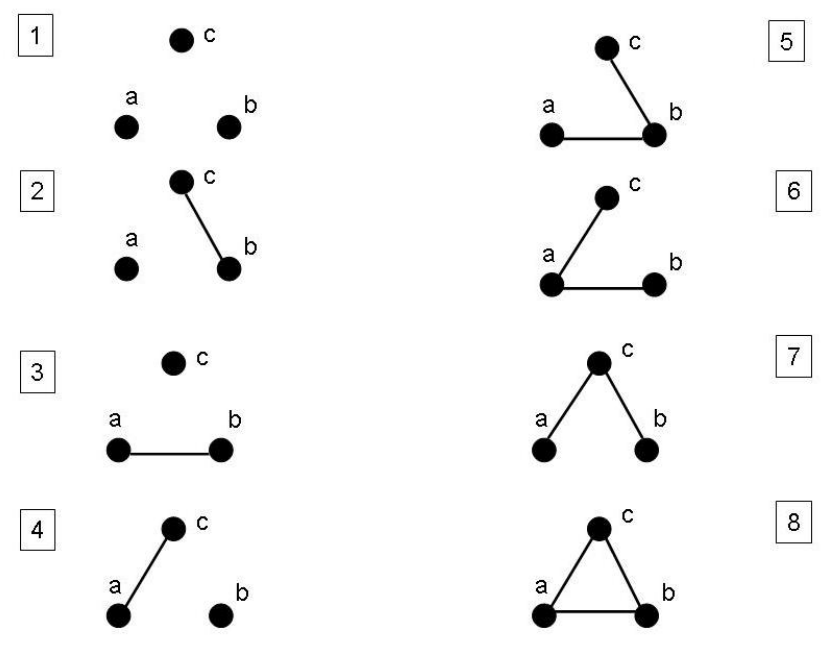

Figure 3. Stabilizer generators of three-partite graph states representing elementary segment of spin network, see Eqs. $(9,10)$

In particular, stabilizers of graph state, given in figure 3 under (7), are represented by the each row of binary matrix $[28,29]$ :

$$
\left(\begin{array}{lll|lll}
0 & 0 & 0 & 0 & 0 & 0 \\
1 & 0 & 0 & 0 & 0 & 1 \\
0 & 1 & 0 & 0 & 0 & 1 \\
0 & 0 & 1 & 1 & 1 & 0 \\
1 & 1 & 0 & 0 & 0 & 0 \\
1 & 0 & 1 & 1 & 1 & 1 \\
0 & 1 & 1 & 1 & 1 & 1 \\
1 & 1 & 1 & 1 & 1 & 0
\end{array}\right)
$$

where nodes of each specified element of the spin network $\{a, b, c\}$, where: $a+b \geqslant c=$ $2 k, a+c \geqslant b=2 k, b+c \geqslant a=2 k$ are encoded in the graph state $(n=3)$ establishing 
incidence relations via following generators:
(1) $\{\{a\},\{b\},\{c\}\} \rightarrow\{|000\rangle\}$,
(2) $\{\{a\}\} \rightarrow\{|000\rangle,|001\rangle,|010\rangle,-|011\rangle\}$,
(3) $\{\{c\}\} \rightarrow\{|000\rangle,|100\rangle,|001\rangle,-|101\rangle\}$
(4) $\{\{b\}\} \rightarrow\{|000\rangle,|010\rangle,|100\rangle,-|110\rangle\}$,
(5) $\{\{a, c\}\} \rightarrow\{|000\rangle,|010\rangle,|101\rangle,|111\rangle\}$,
(6) $\{\{b, c\}\} \rightarrow\{|000\rangle,|100\rangle,|011\rangle,|111\rangle\}$,
(7) $\{\{a, b\}\} \rightarrow\{|000\rangle,|001\rangle,|110\rangle,|111\rangle\}$,
(8) $\{\{a, b, c\}\} \rightarrow\{|100\rangle,|010\rangle,|001\rangle,-|111\rangle\}$,

where (5-7) represent standard 3-qubit flip code on the code subspace: $V_{S}=\{|000\rangle,|111\rangle\}$ for stabilizer set $S=\left\{I, Z_{1} Z_{2}, Z_{2} Z_{3}, Z_{1} Z_{3}\right\}, I=\left(Z_{1} Z_{2}\right)^{2}$.

\section{Conclusion}

We have analyzed implementation of graph states in composing the spin networks architectures. The characterization of graph states is utilized via underlying graph construction defined in terms of affine simplexes with respect to path-connection induced homeomorphisms and polytope construction herein. Future outlook is implementation of higher dimensional homologies in order to establish a self-correcting memory which allows secure data processing [29] without continual active error correction via stabilizer measurement.

\section{References}

[1] Shor P. W. SIAM J. Comput. 26 (5),1484-1509 (1997)

[2] Kak S.C. Advances in Imaging and Electron Physics 94, 259 (1995)

[3] Fei L., Baoyu Z., in Neural Networks and Signal Processing, 2003. Proceedings of the 2003 International Conference on, vol. 1 (IEEE, 2003), vol. 1, pp. 539-542

[4] Töth G., Lent C.S., Tougaw P.D., Brazhnik Y., Weng W., Porod W., Liu R.W., Huang Y.F., arXiv preprint cond-mat/0005038 (2000)

[5] Penrose, R.: Applications of negative dimensional tensors, in Combinatorial Mathematics and its Applications, Academic Press (1971)

[6] Rovelli, C. and Smolin, L. Nucl. Phys. B 331, 80-152 (1990)

[7] Rovelli C. and Smolin, L. Nucl. Phys. B 442, 593-619 (1995)

[8] Seth, M.A. Am. J. Phys. 67(11), 972 (1999)

[9] Baez, J.C. Adv. Math. 117(2), 253 (1996)

[10] Baez, J.C. Journal of functional analysis 158, 253-266 (1998)

[11] Bartolo, C., Di Gambini, R., Griego, J. and Pullin J. Phys. Rev. Lett. 84(11), 2314-2317 (2000)

[12] Grünbaum, B.: Convex Polytopes 2nd edn, New York: Springer (2003) 
[13] Ziegler, G.M.: Lectures on Polytopes, Berlin: Springer (1995)

[14] Whitehead, G.W.: Elements of Homotopy Theory, New York: Springer (1978)

[15] Gray, B.: Homotopy theory. Pure and Appl. Math. 64, New York, Academic Press (1975)

[16] Griffiths, H.B.: The fundamental group of two spaces with a common point. Quart. J. of Math. 5, 175-190 (1954)

[17] Diestel R.: Graph theory, Graduate Texts in Mathematics, Springer-Verlag, Heidelberg, Vol. 173. (2005)

[18] Rosen, K.H.: Handbook of Discrete and Combinatorial Mathematics CRC, Boca Raton, FL (1999)

[19] Seifert, H.: Konstruktion dreidimensionaler geschlossener Räume. Ber. Sächs. Akad. Wiss. 83, 26-66 (1931)

[20] van Kampen, E.H. Amer. J. Math. 55, 261-267 (1933)

[21] Gottesman, D.: Stabilizer codes and quantum error correction, Ph.D. thesis, California Institute of Technology (1997)

[22] Nielsen, M.A. and Chuang I.L.: Quantum Computation and Quantum Information, Cambridge Series on Information and the Natural Sciences, 1st ed., Cambridge University Press, Cambridge (2004)

[23] Hatcher, A.: Algebraic Topology, Cambridge, England: Cambridge University Press (2002)

[24] Berec, V. EPJ Web of Conferences 95, 04007 (2015)

[25] Berec, V. Entropy, 17(5), 3376-3399 (2015)

[26] Goebel, K., Kirk, W.A.: Topics in metric fixed point theory. Cambridge Stud. Adv. Math. 28, Cambridge University Press, 140 (1990)

[27] Gottesman, D. Phys. Rev. A 54, 1862 (1996)

[28] Calderbank, A., Rains, E., Shor, P. and Sloane, N. Phys. Rev. Lett.78, 405 (1997)

[29] Pemberton-Ross, P.J., Kay A. Phys. Rev. Lett. 106(2), 020503 (2011) 